\title{
Detection of the Epstein-Barr virus in blood and bone marrow mononuclear cells of patients with aggressive B-cell non-Hodgkin's lymphoma is not associated with prognosis
}

\author{
HERLANDER MARQUES ${ }^{1,2}$, RAQUEL CATARINO ${ }^{3}$, NELSON DOMINGUES ${ }^{4}$, ELIANE BARROS ${ }^{3}$, \\ CATARINA PORTELA ${ }^{2}$, MARIA INÊS ALMEIDA ${ }^{1}$, SANDRA COSTA ${ }^{1}$, RUI MANUEL REIS ${ }^{1,5}$, \\ RUI MEDEIROS ${ }^{3,6}$ and ADHEMAR LONGATTO-FILHO ${ }^{1,7}$

\begin{abstract}
${ }^{1}$ Life and Health Sciences Research Institute (ICVS), School of Health Sciences, University of Minho;
${ }^{2}$ Department of Oncology, Hospital of Braga, Braga; ${ }^{3}$ Molecular Oncology Group and Virology Laboratory, Portuguese Institute of Oncology; ${ }^{4}$ Oncology-Hematology Department, Institute of Oncology, Porto, Portugal;

${ }^{5}$ Molecular Oncology Research Center, Barretos Cancer Hospital, Barretos, São Paulo, Brazil;

${ }^{6}$ Faculty of Health Sciences of Fernando, Pessoa University, Porto, Portugal;

${ }^{7}$ Laboratory of Medical Investigation (LIM) 14, Faculty of Medicine, University of São Paulo, São Paulo, Brazil
\end{abstract}

Received May 31, 2012; Accepted August 2, 2012

DOI: $10.3892 / \mathrm{ol} .2012 .913$

\begin{abstract}
The Epstein-Barr virus (EBV) is associated with a large spectrum of lymphoproliferative diseases. Traditional methods of EBV detection include the immunohistochemical identification of viral proteins and DNA probes to the viral genome in tumoral tissue. The present study explored the detection of the EBV genome, using the BALF5 gene, in the bone marrow or blood mononuclear cells of patients with diffuse large B-cell lymphomas (DLBCL) and related its presence to the clinical variables and risk factors. The results show that EBV detection in $21.5 \%$ of patients is not associated with age, gender, staging, B symptoms, international prognostic index scores or any analytical parameters, including lactate dehydrogenase (LDH) or $\beta-2$ microglobulin (B2M). The majority of patients were treated with R-CHOP-like (rituximab, cyclophosphamide, doxorubicin, vincristine and prednisolone or an equivalent combination) and some with CHOP-like chemotherapy. Response rates [complete response $(\mathrm{CR})+$ partial response (PR)] were not significantly different between EBV-negative and -positive cases, with 93.2 and $88.9 \%$, respectively. The survival rate was also similar in the two groups, with 5-year overall survival (OS) rates of 64.3 and $76.7 \%$, respectively. However, when analyzing the treatment groups separately there was a trend in EBV-positive patients for
\end{abstract}

Correspondence to: Dr Herlander Marques, Life and Health Sciences Research Institute, School of Health Sciences, University of Minho, Gualtar, Minho, Braga 4710-057, Portugal

E-mail: herlandermarques@hotmail.com

Key words: Epstein-barr virus, non-Hodgkin's lymphoma, diffuse large B-cell lymphoma, mononuclear cells, non-Hodgkin's lymphoma risk factors, rituximab a worse prognosis in patients treated with CHOP-like regimens that was not identified in patients treated with R-CHOP-like regimens. We conclude that $\mathrm{EBV}$ detection in the bone marrow and blood mononuclear cells of DLBC patients has the same frequency of EBV detection on tumoral lymphoma tissue but is not associated with the risk factors, response rate and survival in patients treated mainly with immunochemotherapy plus rituximab. These results also suggest that the addition of rituximab to chemotherapy improves the prognosis associated with EBV detection in DLBCL.

\section{Introduction}

The Epstein-Barr virus (EBV) belongs to the herpes virus family and infects more than $90 \%$ of the human population (1). It is associated with the development of lymphoid tumors and probably also with epithelial neoplasms. EBV colonizes $\mathrm{B}$ cells and can persist as a life-long asymptomatic infection. It has been implicated as the causative agent in a number of lymphoproliferative diseases, including Burkitt's lymphoma, Hodgkin's lymphoma, plasmablastic lymphoma, certain diffuse large B-cell lymphomas (DLBCL) and sub-types of peripheral T-cell lymphomas, including natural killer/T-cell lymphomas, angioimmunoblastic and lymphomatoid granulomatosis (2-4). EBV has also been associated with immunodeficient states such as post-transplant lymphoproliferative disorders (LPDs) and human immunodeficiency virus (HIV)/acquired immunodeficiency syndrome (AIDS)-associated lymphomas $(5,6)$. 'EBV-positive diffuse large B-cell lymphoma of the elderly' has been recognized as a provisional entity of DLBCL. This association was initially described by Oyama $e t a l$ in patients predominantly older than 50 ; however, it has also been documented in younger patients without immunodeficiency (7).

There is presently substantial evidence supporting the etiological role of EBV in cancer. In vitro, the virus trans- 
forms resting B cells into a lymphoblastoid cell line; in vivo, EBV-infected cells do not mature to become a resting memory cell as they are unable to exit the cell cycle. When T-cell function that usually plays a determinant role in controlling EBV-associated lymphoproliferative disease is blocked by immunosuppressive agents or by HIV infection, there is an increased risk of EBV-positive B-cell LPDs $(2,6)$.

$\mathrm{EBV}$ is most frequently detected by immunohistochemistry using antibodies to viral proteins expressed in the cytoplasm and cellular membrane, namely latent membrane protein (LMP) and EB nuclear antigen (EBNA) (7).

However, the standard procedure of EBV diagnosis has been EBV-encoded RNA (EBER) detection by RNA in situ hybridization (RNA-ISH) in tumor tissue. EBER is consistently and highly expressed in latent infection and its detection has a high diagnostic sensitivity for EBV-positive lymphomas (8). PCR-based techniques of genomic isolates obtained from tumor tissue substitutes RNA-ISH with similar sensitivity and specificity and are also useful for strain determination in EBV types 1 or $2(9)$.

Few studies have investigated EBV tumoral detection and prognosis in DLBCL. Three published studies, from Japanese, Peruvian and Korean populations with patient survival analyses, demonstrated the prognostic value of EBV expression using various methods. Generally, EBV-positive lymphomas are associated with a worse overall survival rate (OS) and are independent of international prognostic index (IPI) scores $(7,10,11)$. Another study, comparing the expression of LMP-1 with various biological parameters in patients predominantly treated with conventional chemotherapy without rituximab, revealed that viral protein is the most potent prognostic factor associated with a poor survival rate (12).

The influence of EBV positivity on the treatment response of patients with DLBCL has also been evaluated. Studies with nodal lymphomas associate the detection of EBV with a worse overall response rate, and one study of extranodal lymphomas revealed that $3 / 4$ cases of gastric DLBCL patients had poor responses $(7,11,13)$. Data concerning the influence of EBV presence in the prognosis and response to treatment of patients with DLBCL are insufficient or incompletely explored and have not been evaluated regarding immune therapy with monoclonal antibodies.

However, it is possible to detect the EBV genome in the peripheral blood of healthy seropositive individuals, although it is usually present in extremely rare latently infected memory B cells. By contrast, the viral load of mononuclear blood cells of EBV-related lymphoproliferative diseases may be high $(14,15)$. The incidence of EBV in DLBCL varies across the world, with Asian, Latin American and Western patients' positivity between 5 and $15 \%$. In Portugal, the incidence of EBV detection among non-Hodgkin's lymphoma (NHL) patients or in the general population is not known.

The detection of EBV proteins or genes is usually performed on tumoral lymphoma tissue and this determines the clinical prognostic impact. The search for and detection of the EBV genome in the blood or bone marrow mononuclear cells of patients with DLBCL and its correlation with prognosis has not been previously reported. The presence of EBV in these cells may be interpreted as circulating colonized tumoral cells or as colonized physiological memory B cells.
The present study sought to investigate the presence of the EBV genome in the blood or bone marrow mononuclear cells of patients with DLBCL, some treated with anthracycline-based chemotherapy alone, others treated with same type of chemotherapy plus rituximab. The prognosis and response according to the patient's type of treatment was also evaluated.

\section{Patients and methods}

Case selection and patients samples. A total of 130 patients aged $>18$ years old and diagnosed with DLBCL in the oncological services of the Portuguese public health system of the cities of Porto and Braga were included, regardless of their serological positivity for EBV. Patients with DLBCL were selected from a pool of 350 with various NHL histological subtypes, sequentially diagnosed and that had had bone marrow and/or blood collected during staging procedures. The Hospital Ethics Committees approved the study and all patients provided written informed consent.

Histological diagnosis was obtained according to the OMS classification (16). Samples, including blood and/or bone marrow aspirates, were collected from all patients. In 121 patients, both blood and bone marrow were collected, in 4 only bone marrow was obtained and in 5 others, only blood was collected. Bone marrow was preferentially used in this study. Mononuclear cells from blood or bone marrow were isolated by centrifugation on Ficoll-Hipac and obtained from the cell-enriched fluid retained in the interface.

Registries of clinical data included demographic variables, Ann Arbor staging system, ECOG Performance Status scale, IPI type of chemotherapy and response to treatment.

DNA preparation. Genetic material was obtained from isolated mononuclear cells by the following method. DNA was extracted using a QIAamp DNA Mini kit (Qiagen, Hilden, Germany) according to the manufacturer's instructions. Purified DNA was eluted in $70 \mu 1$ of buffer AE for elution and stored at $-20^{\circ} \mathrm{C}$.

Virus DNA detection. The identification of EBV DNA was performed by the real-time quantitative PCR method, based on a continuous monitoring of fluorescence by an optical system (17). The probe was labeled by two fluorescent dyes. One served as a reporter on the 50 end (VIC dye; Applied Biosystems, Foster City, CA, USA). The emission spectrum of the dye was quenched by a second fluorescent dye at the 30 end (TAMRA; Applied Biosystems). The primer and probe sequences were selected from BALF5 gene codifying the DNA polymerase of EBV as following: forward primer, 5'-CGGAAGCCCTCTGGACTTC-3'; reverse, 5'-CCCTGT TTATCCGATGGAATG-3'. A fluorogenic probe, 5'-TGTACA CGCACGAGAAATGCGCC-3', with a sequence localized between PCR primers was obtained from Applied Biosystems.

Fluorogenic PCRs were carried out in a reaction volume of $25 \mu \mathrm{l}$ in a 7300 ABIPRISM System (Applied Biosystems). Each real-time PCR reaction consisted of TaqMan Universal Mastermix (Applied Biosystems), probe $(7.5 \mathrm{mmol} / \mathrm{l})$, forward primer $(5 \mathrm{mmol} / \mathrm{l})$, reverse primer $(5 \mathrm{mmol} / \mathrm{l})$ and DNA solution $(2.5 \mu \mathrm{l})$. Thermal cycling was initiated with a denaturation step of $50^{\circ} \mathrm{C}$ for $2 \mathrm{~min}$ and then $95^{\circ} \mathrm{C}$ for $10 \mathrm{~min}$ followed by 
Table I. Prognostic factors for all populations and for EVB-positive and -negative subgroups.

\begin{tabular}{|c|c|c|c|c|}
\hline Variable & Frequency & EVB-negative & EBV-positive & P-value \\
\hline $\mathrm{n}$ & 130 & 102 & 28 & \\
\hline Median age (years) & 57.9 & 57.5 & 59.3 & \\
\hline$>60$ years old, $\mathrm{n}(\%)$ & $65(50)$ & $50(49)$ & $15(53.6)$ & 0.67 \\
\hline Gender (male), n (\%) & $73(56.2)$ & $58(56.9)$ & $15(53.6)$ & 0.76 \\
\hline Primary extra-nodal $^{a}$ & $43 / 129(33.3 \%)$ & $34(33.3 \%)$ & $9 / 27(33.3 \%)$ & 1 \\
\hline Ann Arbor stage III/IV ${ }^{\mathrm{a}}$ & $58 / 128(45.3 \%)$ & $48(47.5 \%)$ & $10(37 \%)$ & 0.33 \\
\hline B symptoms ${ }^{\mathrm{a}}$ & $60 / 127(47.2 \%)$ & $48(48 \%)$ & $12(44.4 \%)$ & 0.74 \\
\hline Performance status $\geq 2^{\text {a }}$ & $32 / 124(25.8 \%)$ & $23(23.7 \%)$ & $9(33.3 \%)$ & 0.31 \\
\hline Elevated B2M ${ }^{\mathrm{a}}$ & $33 / 101(32.7 \%)$ & $23(29.5 \%)$ & $10(43.5 \%)$ & 0.21 \\
\hline Elevated $\mathrm{LDH}^{\mathrm{a}}$ & $66 / 124(53.2 \%)$ & $48(49 \%)$ & $18(69.2 \%)$ & 0.07 \\
\hline IPI IH+H ${ }^{a}$ & $35 / 124(28.2 \%)$ & $28(28.6 \%)$ & $7(26.9 \%)$ & 0.86 \\
\hline Treatment response $\geq \mathrm{PR}^{\mathrm{a}}$ & $108 / 119(90.8 \%)$ & $84(93.2 \%)$ & $24(88.9 \%)$ & 0.90 \\
\hline Relapsed $^{\mathrm{a}}$ & $16 / 74(21.6 \%)$ & $15(26.3 \%)$ & $1(5.9 \%)$ & 0.07 \\
\hline 4-year PFS ${ }^{\mathrm{b}}, \%$ & 71.6 & 70.2 & 82.9 & 0.69 \\
\hline 5-year survival ${ }^{\mathrm{b}}, \%$ & 65.2 & 64.3 & 76.7 & 0.91 \\
\hline
\end{tabular}

${ }^{a}$ Missing data; ${ }^{b} \log$ rank test. EBV, Epstein-Barr virus; B2M, $\beta$-2 microglobulin; LDH, lactate dehydrogenase; IPI, international prognostic index; IH, intermediate-high; H, high; PR, partial remission; PFS, progression-free survival.

50 cycles of denaturation at $95^{\circ} \mathrm{C}$ for $15 \mathrm{sec}$, annealing and extension at $60^{\circ} \mathrm{C}$ for $1 \mathrm{~min}$. Each plate consisted of patient samples in duplicate and triplicate blanks of water as a negative control. The calibration curve of each plate was calculated based on a dilution series of the TaqMan Control Human Genomic DNA Standard (Applied Biosystems) from 1 to $1 \times 10^{-3} \mathrm{ng} / \mathrm{ml}$ at $10 \mathrm{ng} / \mu \mathrm{l}: 1,0.1,0.01,0.001$ and $0.0001 \mathrm{ng} / \mathrm{ml}$. All data were analyzed using the 7300 System-SDS Software (version 1.2.3) Sequence Detection Software (Applied Biosystems).

\section{Results}

Diagnostic features. A total of 130 patients diagnosed with DLBCL were selected for this study. The median age was 58 years and $56 \%$ were male. In total, 127 patients were diagnosed as DLBC and 3 as Burkitt-like NHL. Of all the cases, $33 \%$ were classified as extra-nodal, $8 \%$ had $>1$ secondary extra-nodal invasion, $47 \%$ had B symptoms, $45 \%$ had advanced stage disease (III and IV), $25.8 \%$ had an ECOG performance status $\geq 2,53 \%$ had elevated lactate dehydrogenase (LDH) levels, $28.2 \%$ had IPI intermediate-high $(\mathrm{IH})$ or high $(\mathrm{H})$ and $32.7 \%$ had elevated $\beta-2$ microglobulin (B2M) levels. A minority of patients $(30 \%)$ received cyclophosphamide, doxorubicin, vincristine and prednisolone $(\mathrm{CHOP})$ or an equivalent combination (CHOP-like) chemotherapy and $60 \%$ received the same chemotherapy combined with rituximab (R-CHOP) or R-CHOP-like. The remaining patients were treated with chemotherapy combinations without anthracyclines. Certain patients were treated before 2003, the year that rituximab was introduced in Portugal.

EBV detection. The EBV genome was detected in 28/130 patients $(21.5 \%)$ in blood and/or bone morrow samples. Bone marrow was preferably used, but for 5 patients only blood samples were available. In another 10, the two samples were tested and were accordant except one case. The characteristics of the EBV-negative and -positive patients are shown in Table I.

Compared with EBV-negative patients, EBV-positive patients had a similar mean age (57.5 versus 59.3 years), gender, nodal or extra-nodal presentation, Ann Arbour staging, IPI risk factors, serum LDH and B2M level, response to treatment, progressionfree survival and OS. Response rate [complete response (CR) + partial response (PR)] was 93.2 and $88.9 \%$ for EBV-negative and -positive patients, respectively (not significant).

The global survival rate was $65 \%$ at 5 years. There was a significant difference at 5 years of survival in the CHOP/ CHOP-like and R-CHOP/R-CHOP-like groups, with 47.7 and $88.6 \%$, respectively $(\mathrm{P}=0.001)$. Globally there was no difference between EBV-positive and -negative cases, but when a Breslow test was used to compare EBV-positive patients treated with CHOP-like or R-CHOP-like chemotherapy, a trend $(\mathrm{P}=0.051)$ favoring R-CHOP-like was attained (Fig. 1).

Clinical variables, treatment response rate and survival was not significantly different in EB-positive and -negative groups (Table I). Fig. 1 shows the survival curves by EBV status and type of chemotherapy. Univariate analysis revealed that age, Ann Arbor stage, B symptoms, ECOG system performance status and LDH and serum albumin levels anticipated a prognostic factor value, independent of the type of treatment, chemotherapy with or without rituximab and EBV status (Table I). When considering IPI in these series, a good discrimination in prognosis of the groups was observed but there was an asymmetrical distribution of the population, with $12 \%$ of patients having a score of $\mathrm{H}$. Fig. 2 shows the survival curves for the IPI groups.

Four cases of DLBCL were diagnosed in patients with AIDS. AIDS diagnosis occurred between 3.5 years and 4 months before the diagnosis of DLBCL and 3 of them were EBV positive Of these patients, 2 succumbed; one from an infectious complication during treatment and the other had 
A

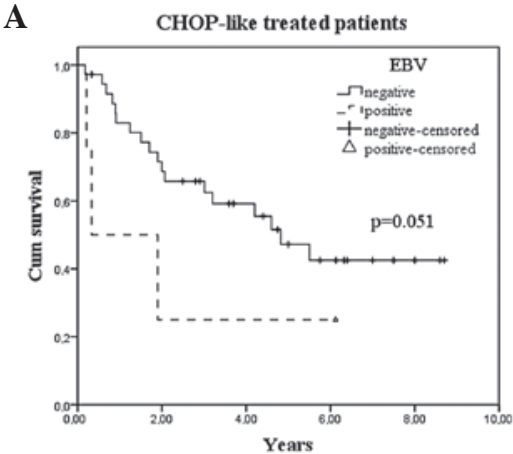

$\mathbf{B}$

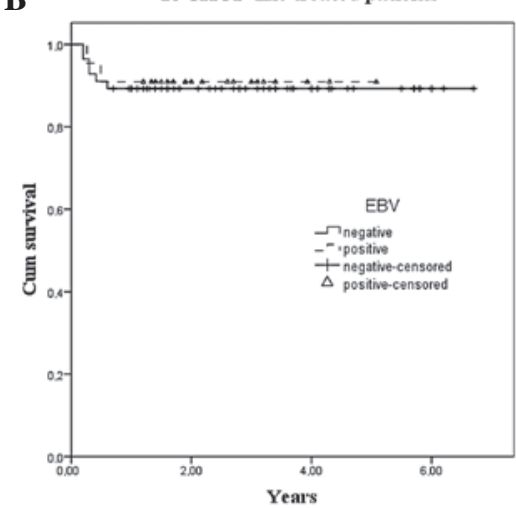

Figure 1. Survival probability according to EBV status and type of treatment. (A) Kaplan-Meier overall survival for patients treated with anthracycline or anthracenedione chemotherapy without the anti-CD20 monoclonal antibody rituximab. (B) Kaplan-Meier overall survival for patients treated with antracycline- or anthracenedione-containing chemotherapy with rituximab. The difference suggests that the combination of rituximab and standard CHOP therapy overcomes a worse prognosis induced by EBV presence on the B cells of patients with DLBCL. OS, overall survival; DLBCL, diffuse large B-cell lymphoma; EBV, Epstein-Barr virus; CHOP, cyclophosphamide, doxorubicin, vincristine and prednisolone; R-CHOP, CHOP with rituximab.

no response to 3 different lines of immune-chemotherapy and succumbed to the disease within 6 months.

\section{Discussion}

There is a limited number of studies investigating EBV tumoral detection and prognosis in DLBCL. To the best of our knowledge, none of these explore the virus presence in mononuclear cells of the blood or bone marrow while also evaluating the prognostic endpoints $(7,10,11)$. The current study evaluated the correlation between the EBV genome expression in mononuclear cells of the bone marrow or peripheral blood in patients with DLBCL with response to treatment and survival.

Noteworthy data have emerged from a Japanese study (7) that evaluated the presence of EBER in tumor tissue, comparing a group of EBV-positive with a group of EBV-negative elderly DLBCL patients. A shorter survival was observed in EBV-positive patients with a median OS of 24 months versus OS not reached in EBV-negative patients, which was a prognostic factor independent of IPI scores. Similar results have been reported in a Peruvian population (10) treated with chemotherapy without rituximab with an OS of 7 and 47 months for EBV-positive and -negative DLBCL patients,

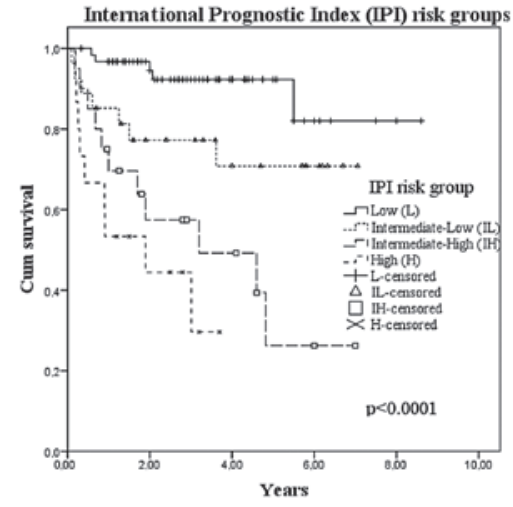

Figure 2: Kaplan-Meier OS curves. Outcome according to the IPI groups. Relative frequency predominance in low risk group may be due to a predominance of localized Ann Arbour stages and a younger median age. OS, overall survival; IPI, international prognostic index.

respectively. In this study, EBER tumoral expression and IPI scores were independent prognostic factors in a multivariate analysis. A study of Korean patients (11) treated with chemotherapy with only a small percentage combined with rituximab also demonstrated that EBV expression was associated with a worsened OS. This association is maintained in sub-groups of high IPI scores and phenotypical non-Germinal Center DLBCL patients. The differences between patients treated with a chemotherapy combination containing rituximab and those treated with a chemotherapy not containing rituximab were not analyzed, likely due to a small number of patients receiving this monoclonal antibody.

In a different approach, Paydas et al (12) evaluated the prognostic significance of biological factors correlated with angiogenic, anti-apoptotic, inflammatory and viral process in 88 DLBCL patients and determined that the expression of LMP-1 is the most important factor to determine the survival rate. Contrasting with those studies, the results of the current study using bone marrow and blood, but not tumoral tissue, revealed no significant difference in survival between EBV-positive and -negative patients.

Cases of EBV-positive aggressive DLBCL have been described among Caucasian populations but a survival analysis has not been reported $(18,19)$. We observed a case with an especially aggressive course in a patient co-infected with EBV and HIV.

The influence of EBV positivity in treatment response on patients with DLBCL has been evaluated by a limited number of studies. Oyama et al compared 62 EBV-positive Japanese patients treated with anthracycline-containing regimens without rituximab with 104 EBV-negative patients and observed overall response rates of 80 and $99 \%$, respectively $(\mathrm{P}<0.0001)$ with $\mathrm{CR}$ rates of 66 and $91 \%$ (7). Park et al reported an overall response rate but not a complete remission rate of 25 EBV-positive and $207 \mathrm{EBV}$-negative patients; the authors identified a significant difference of 72 and $92 \%$, respectively (11). In a gastric DLBCL study, 4 EBV-positive patients had a poor response to chemotherapy and radiotherapy with 2 of those presenting with progressive disease, one partial and one complete remission (13).

The search for EBV presence in mononuclear cells in bone morrow and/or blood and the evaluation of its value 
as a prognostic tool has not been systematically performed. In a Brazilian study of children with Burkitt's lymphoma comparing different methods of EBV detection, only 9 cases were simultaneousely tested in tumor mass samples and bone marrow (9). The authors searched clonal lymphoma cells by immunoglobulin $\mathrm{H}$ gene rearrangement. They found that only EBV-tumor cell-positive cases with bone marrow clonal infiltrating cells were also EBV-positive. However, risk factors were not evaluated in this study.

The present study was conducted on blood and bone marrow searching for the presence of EBV in the mononuclear cells of these tissues and aimed to evaluate the prognostic relevance of the virus in patients with DLBCL. With a sensitive technology such as PCR it was possible to detect EBV-positive memory cells, non-tumoral lymphocytes and true malignant lymphocytes. However, the frequency of colonized cells in peripheral blood is very rare in healthy seropositive individuals, with an count under $1 / 50,000$ in the majority of studies $(14,15)$. These findings were the basis of the present study.

When comparing the results of the present study using bone marrow/blood samples with studies performed elsewhere that used tumor tissue cells, the frequency of detection is not substantially different, with 21.5 and $5-15 \%$, respectively. This frequency is higher than that published for the western population, but it included 4 cases of HIV-positive patients. This result also indicates that searching in bone marrow or blood mononuclear cells may be a reliable method of EBV detection. However, it cannot distinguish tumor B cells from the rare EBV-colonized memory B cells, either in circulation or in bone marrow. Therefore, we plan to search for EBV on tissue cells and to compare the results between bone marrow and blood in the same patient.

In contrast to the results of others, the negative prognostic impact usually observed in DLBCL has not been verified in the current study. This may be explained by the introduction of rituximab in therapeutic armamentarium to B-cell NHL. The majority of patients received $\mathrm{R}-\mathrm{CHOP}$ and some were administered dose-densified R-CHOP-14, which had not occurred in the majority of the patients in previous studies. In fact, the prognostic influence of EBV presence in the rituximab era remains unknown. The addition of rituximab to $\mathrm{CHOP}$ chemotherapy may overcome the prognostis of $\mathrm{Bcl} 2$ expression on DLBCL in patients treated with $\mathrm{CHOP}$ and an identical influence may be possible on EBV expression (20). Favoring this hypothesis is the efficacy of rituximab in post-transplant lymphoproliferative disease, a syndrome typically associated with EBV and immunodepression (21). The current study also supports the favorable influence of rituximab, given that when CHOP-like versus R-CHOP-like patients were analyzed, the trend of a decreased survival rate in EBV-positive patients was only observed in the first group, whose treatment did not include rituximab.

In conclusion, this study demonstrated the presence of a high percentage of EBV in mononuclear cells from bone marrow aspirates or the peripheral blood of patients with DLBCL. Detection of the virus genome was not associated with any clinical variable, response rate or survival rate, regardless of treatment, but the majority of patients in this study received chemotherapy and rituximab. Moreover, we provide evidence to support the use of rituximab in addition to conventional chemotherapy to improve the clinical outcomes associated with EBV detection in DLBCL.

\section{References}

1. Cohen JI: Epstein-Barr virus infection. N Engl J Med 343: 481-492, 2000

2. Thorley-Lawson DA and Gross A: Persistence of the Epstein-Barr virus and the origins of associated lymphomas. N Engl J Med 350: 1328-1337, 2004

3. Kelly G, Bell A and Rickinson A: Epstein-Barr virus-associated Burkitt lymphomagensis selects for downregulation of the nuclear antigen EBNA2. Nat Med 8: 1098-1104, 2002.

4. Banks PM and Warnke RA: Mature T-cell and NK-cell neoplasms. In: WHO Classification of Tumors: Pathology and Genetics of Tumours of Hematopoetic and Lymphoid Tissues. Jaffe ES, Harris NL, Stein H and Vardiman JW (eds). IARC Press, Lyon, pp189-230, 2001.

5. Kuzushima K, Kimura H, Hoshino Y, et al: Longitudinal dynamics of Epstein-Barr virus-specific cy totoxic T lymphocytes during posttransplant lymphoproliferative disorder. J Infect Dis 182: 937-940, 2000.

6. Hamilton-Dutoit SJ, Raphael M, Audouin J, et al: In situ demonstration of Epstein-Barr virus small RNAs (EBER 1) in acquired immunodeficiency syndrome-related lymphomas: correlation with tumor morphology and primary site. Blood 82: 619-624, 1993.

7. Oyama T, Yamamoto $\mathrm{K}$, Asano $\mathrm{N}$, et al: Age-related EBV-associated B-cell lymphoproliferative disorders constitute a distinct clinicopathologic group: a study of 96 patients. Clin Cancer Res 13: 5124-5132, 2007.

8. Ambinder RF and Mann RB: Detection and characterization of Epstein-Barr virus in clinical specimens. Am J Pathol 145: 239-252, 1994.

9. Hassan R, White LR, Stefanoff CG, et al: Epstein-Barr (EBV) detection and typing by PCR: a contribution to diagnostic screening of EBV-positive Burkitt's lymphoma. Diagn Pathol 1: 17-23, 2006.

10. Morales D, Beltran B, De Mendoza FH, et al: Epstein-Barr virus as a prognostic factor in de novo nodal diffuse large B-cell lymphoma. Leuk Lymphoma 51: 66-72, 2010.

11. Park S, Lee J, Ko YH, et al: The impact of Epstein-Barr virus status on clinical outcome in diffuse large B-cell lymphoma. Blood 110: 972-978, 2007.

12. Paydas S, Ergin M, Seydaoglu G, et al: Prognostic significance of angiogenic/lymphangiogenic, anti-apoptotic, inflammatory and viral factors in 88 cases with diffuse large B cell lymphoma and review of the literature. Leuk Res 33: 4117-4126, 2009.

13. Yoshino T, Nakamura S, Matsuno Y, et al: Epstein-Barr virus involvement is a preditive factor for the resistance to chemoradiotherapy of gastric diffuse large B-cell lymphoma. Cancer Sci 97: 163-166, 2006

14. Tierney RJ, Steven N, Young LS and Rickinson AB: Epstein-Barr virus latency in blood mononuclear cells: Analysis of viral gene transcription during primary infection and in the carrier state. $\mathrm{J}$ Virol 68: 7374-7385, 1994.

15. Decker LL, Klaman LD and Thorley-Lawson DA: Detection of the latent form of Epstein-Barr virus DNA in peripheral blood of healthy individuals. J Virol 70: 3286-3299, 1996.

16. Swerdlow SH, Campo E, Harris NE, et al (eds): WHO Classification of Tumours of Haematopoietic and Lymphoid Tissue. 4th edition. IARC Press, Lyon, 2008.

17. Heid CA, Stevens J, Livak KJ and Williams PM: Real time quantitative PCR. Genome Res 6: 986-994, 1996.

18. Gibson SE and Hsi ED: Epstein-Barr virus-positive B-cell lymphoma of elderly at a United States tertiary medical center: an uncommon aggressive lymphoma with a nongerminal center B-cell phenotype. Hum Pathol 40: 653-661, 2009.

19. Hoeller S, Tzankov A, Pileri SA, et al: Epstein-Barr virus-positive diffuse large B cell lymphoma in elderly patients is rare in Western populations. Hum Pathol 41: 352-357, 2010.

20. Mounier N, Briere J, Gisselbrecht C, et al: Rituximab plus CHOP (R-CHOP) overcomes bcl-2-associated resistance to chemotherapy in elderly patients with diffuse large B-cell lymphoma (DLBCL). Blood 101: 4279-4284, 2003.

21. Milpied N, Vasseur B, Parquet N, et al: Humanized anti-CD20 monoclonal antibody (Rituximab) in post transplant B-lymphoproliferative disorder: A retrospective analysis on 32 patients. Ann Oncol 11: 113-116, 2000. 